\title{
Full Spectrum of the Liouvillian of Open Dissipative Quantum Systems in the Zeno Limit
}

\author{
Vladislav Popkov ${ }^{1}$ and Carlo Presilla $\oplus^{2,3}$ \\ ${ }^{1}$ Department of Physics, University of Wuppertal, Gausstraße 20, 42119 Wuppertal, Germany \\ ${ }^{2}$ Dipartimento di Fisica, Sapienza Università di Roma, Piazzale Aldo Moro 2, Roma 00185, Italy \\ ${ }^{3}$ Istituto Nazionale di Fisica Nucleare, Sezione di Roma 1, Roma 00185, Italy
}

(Received 13 January 2021; accepted 12 April 2021; published 11 May 2021)

\begin{abstract}
We consider an open quantum system with dissipation, described by a Lindblad Master equation (LME). For dissipation locally acting and sufficiently strong, a separation of the relaxation timescales occurs, which, in terms of the eigenvalues of the Liouvillian, implies a grouping of the latter in distinct vertical stripes in the complex plane at positions determined by the eigenvalues of the dissipator. We derive effective LME equations describing the modes within each stripe separately, and solve them perturbatively, obtaining for the full set of eigenvalues and eigenstates of the Liouvillian explicit expressions correct at order $1 / \Gamma$ included, where $\Gamma$ is the strength of the dissipation. As an example, we apply our general results to quantum $X Y Z$ spin chains coupled, at one boundary, to a dissipative bath of polarization.
\end{abstract}

DOI: 10.1103/PhysRevLett.126.190402

Recently a great deal of analytic progress has been made in the theory of open quantum systems and their steadystate exact solutions. Much less is known about the full spectrum of the Liouvillian [the Lindbladian, more precisely, if the open quantum system is described, as very often happens, by a Lindblad master equation (LME)]. Just to say, the knowledge of this spectrum is essential to predict the finite-time evolution of dissipative systems, as of interest in fields ranging from quantum computing [1] to quantum biology [2]. The problem basically remains intractable, except via hard computational methods [3-6].

The existing literature regarding the Liouvillian general properties focuses on an analysis of asymptotic time regime $t \rightarrow \infty$, i.e., putting emphasis on the existence of a decoherence-free subspace and the asymptotic leakage out of it [7-9]. Within such an approach, however, a substantial part of information about the Liouvillian spectrum is lost.

Exceptionally, under special conditions imposed on the Lindblad operators and the Hamiltonian, the Liouvillian spectrum can be related to the spectrum of auxiliary nonHermitian operators. However, even in this case, the complete set of eigenstates is out of reach [10-13].

In the present communication, in contrast, we show how to obtain the complete set of eigenvalues and eigenstates of the Liouvillian, provided that the dissipation is sufficiently strong with respect to the coherent part of the evolution, in the so-called quantum Zeno regime [14-17]. For this setup to be nontrivial, dissipation must act only on a part of the degrees of freedom.

As we will see, in the limit of strong dissipation acting on a part of degrees of freedom, the behavior of an open quantum system simplifies and the full Liouvillian can be block diagonalized.
We provide a general procedure to obtain the full set of eigenvalues and eigenstates by means of a perturbative approach in terms of the solution of a linear problem for the dissipation-projected Hamiltonian [18,19], and other related Hamiltonians acting in a reduced Hilbert space. As an example, we comprehensively discuss the case of general open $X Y Z$ spin chains with arbitrary spin states targeted at one of the boundaries by the strong interaction with dissipative environments.

General theory.-We consider an open quantum system with finite Hilbert space $\mathcal{H}$ and dissipation acting only on a part of its degrees of freedom, namely, those associated to the subspace $\mathcal{H}_{0} \subset \mathcal{H}$. Denoting by $\mathcal{H}_{1}$ the dissipation-free subspace, we have $\mathcal{H}=\mathcal{H}_{0} \otimes \mathcal{H}_{1}$ with $\operatorname{dim} \mathcal{H}_{0}=d_{0}, \operatorname{dim} \mathcal{H}_{1}=d_{1}$, and $d_{0} d_{1}=d=\operatorname{dim} \mathcal{H}$. The evolution of the reduced density matrix operator of the systems, $d \rho(\tau) / d \tau=\mathcal{L}[\rho(\tau)]$, is determined by the Liouvillian

$$
\mathcal{L}[\cdot]=-i[H, \cdot]+\Gamma \mathcal{D}[\cdot],
$$

where $H$ is the Hamiltonian of the system, $\mathcal{D}[\cdot]$ a Lindblad dissipator of standard form, and $\Gamma$ the strength of the dissipation. The use of a Markovian Lindblad dynamics for large dissipation is justified for reservoirs with very short correlation times [20]. Note that we work in units of $\hbar=1$, i.e., $\tau=t_{\mathrm{ph}} / \hbar$ and $\Gamma=\Gamma_{\mathrm{ph}} \hbar$, where $t_{\mathrm{ph}}$ and $\Gamma_{\mathrm{ph}}$ are the physical time and dissipation strength.

In Ref. [18] it has been shown that in the Zeno limit $\Gamma \rightarrow \infty$ the dynamics (1) is still reduced to a new Lindblad equation written in terms of a renormalized Hamiltonian and an effective dissipator. More precisely, for times $\tau \gg 1 / \Gamma$ and with an error $O\left(1 / \Gamma^{2}\right)$ we have $\rho(\tau)=\psi_{0} \otimes R_{0}(\tau)$, where $\psi_{0} \in \mathcal{H}_{0}$ is the dissipator 
kernel, $\mathcal{D}\left[\psi_{0}\right]=0$, (assumed to be unique) and $R_{0}(\tau) \in \mathcal{H}_{1}$ satisfies

$$
\frac{d R_{0}(\tau)}{d \tau}=-i\left[h_{D}+\tilde{H}_{a} / \Gamma, R_{0}(\tau)\right]+\frac{1}{\Gamma} \tilde{\mathcal{D}}\left[R_{0}(\tau)\right] .
$$

The effective Hamiltonian $\tilde{H}=h_{D}+\tilde{H}_{a} / \Gamma \quad$ is the sum of the dissipation-projected Hamiltonian, $h_{D}=\operatorname{tr}_{\mathcal{H}_{0}}\left[\left(\psi_{0} \otimes I_{\mathcal{H}_{1}}\right) H\right]$, and a Lamb shift correction $\tilde{H}_{a}$. With $\operatorname{tr}_{\mathcal{H}_{0}}$ we indicate the trace in the subspace $\mathcal{H}_{0}$. Note that both $\tilde{H}_{a}$ and the effective dissipator $\tilde{\mathcal{D}}[\cdot]$ act in the sole subspace $\mathcal{H}_{1}$. Explicit expressions of $\tilde{H}_{a}$ and $\tilde{\mathcal{D}}[\cdot]$ are given in [6] and, for convenience, reported in Supplemental Material [21].

Equation (2) provides complete information about $R_{0}$, the dissipation-free component of the density matrix $\rho$. The full density matrix has, however, an expansion of the form $\rho(\tau)=\sum_{k} \psi_{k} \otimes R_{k}(\tau)$, where $\psi_{k}$ are the eigenstates of the original dissipator $\mathcal{D}$ (which we assume diagonalizable),

$$
\mathcal{D}\left[\psi_{k}\right]=c_{k} \psi_{k} .
$$

The complex eigenvalues $c_{k}$ always have a nonpositive real part and one of them is 0 , conventionally, $c_{0}=0$. When $\Gamma$ is large, all the components $k>0$ of the density matrix lying outside the dissipation-free subspace can be shown to scale as $1 / \Gamma$, namely, $\left\|R_{k}(\tau)\right\|=O(1 / \Gamma)$ for $\tau>O(1)$, see [19].

The spectrum of the effective Liouvillian $\tilde{\mathcal{L}}$ associated to Eq. (2) gives only a part of the full Liouvillian spectrum, namely, $d_{1}^{2}$ eigenvalues out of $d^{2}=\left(d_{0} d_{1}\right)^{2}$. The remaining $d^{2}-d_{1}^{2}$ eigenvalues of the Liouvillian $\mathcal{L}$ originate from the components $R_{k}$ with $k>0$ in the expansion of the full density matrix.

In [18] it has been shown how to obtain, in the Zeno limit, the nonequilibrium steady state, i.e., the eigenstate of $\mathcal{L}$ corresponding to the eigenvalue 0 . Here, we derive explicit formulas for all the eigenvalues and eigenstates of $\mathcal{L}$ near the Zeno limit, up to order $1 / \Gamma$ included. Explicitly, we will first obtain equations analogous to Eq. (2) for all the components $R_{k}(\tau)$ of the density matrix, and then show how to use these equations to derive eigenvalues and eigenstates of $\mathcal{L}$.

In order to formulate our main statement, note that the dissipator eigenstates $\left\{\psi_{k}\right\}$ of Eq. (3) form a basis in $\mathcal{H}_{0}$. Let $\left\{\varphi_{k}\right\}$ be a biorthogonal basis in $\mathcal{H}_{0}$ satisfying $\operatorname{tr}\left(\psi_{k} \varphi_{n}\right)=\delta_{k n}$. The decompositions of the Hamiltonian $H$ and of the density matrix $\rho(\tau)$ in the bases $\left\{\varphi_{k}\right\}$ and $\left\{\psi_{k}\right\}$ are, respectively,

$$
\begin{aligned}
H & =\sum_{m}\left(\varphi_{m}^{\dagger} \otimes g_{m}^{\dagger}\right)=\sum_{m}\left(\varphi_{m} \otimes g_{m}\right), \\
g_{m} & =\operatorname{tr}_{\mathcal{H}_{0}}\left[\left(\psi_{m} \otimes I_{\mathcal{H}_{1}}\right) H\right],
\end{aligned}
$$

$$
\begin{aligned}
\rho(\tau) & =\sum_{k} \psi_{k} \otimes R_{k}(\tau), \\
R_{k}(\tau) & =\operatorname{tr}_{\mathcal{H}_{0}}\left[\left(\varphi_{k} \otimes I_{\mathcal{H}_{1}}\right) \rho(\tau)\right] .
\end{aligned}
$$

Statement.-The component $R_{k}$ corresponding to a nonzero dissipator eigenvalue $c_{k}$ with degeneracy deg, near the Zeno limit satisfy

$$
\begin{aligned}
\frac{d R_{k}}{d \tau}= & \Gamma c_{k} R_{k}+i \sum_{s: c_{s}=c_{k}}\left(U_{k, s} R_{s}-R_{s} W_{k, s}\right) \\
& +\frac{1}{\Gamma} \sum_{z>0} \sum_{m>0} \sum_{n: c_{n} \neq c_{k}} \sum_{s: c_{s}=c_{k}} \frac{1}{c_{n}-c_{k}} \\
& \times\left(-\gamma_{m, z}^{n, s, k} g_{m} R_{s} g_{z}^{\dagger}+\varepsilon_{z, m}^{n, s, k} g_{z}^{\dagger} g_{m} R_{s}+\delta_{z, m}^{n, s, k} R_{s} g_{z}^{\dagger} g_{m}\right) \\
& +O\left(1 / \Gamma^{2}\right)
\end{aligned}
$$

where $U_{k, s}$ and $W_{k, s}$ are operators in $\mathcal{H}_{1}$ given by

$$
U_{k, s}=\sum_{n} B_{n, s, k} g_{n}^{\dagger}, \quad W_{k, s}=\sum_{n} A_{n, s, k} g_{n}^{\dagger},
$$

and $\gamma_{m, z}^{n, s, k}, \varepsilon_{m, z}^{n, s, k}$, and $\delta_{m, z}^{n, s, k}$ are the coefficients

$$
\begin{aligned}
\gamma_{m, z}^{n, s, k} & =C_{m, s, n} A_{z, n, k}+A_{z, s, n} C_{m, n, k}, \\
\epsilon_{z, m}^{n, s, k} & =C_{m, s, n} B_{z, n, k}, \\
\delta_{z, m}^{n, s, k} & =A_{z, s, n} C_{k, n, m}
\end{aligned}
$$

with

$$
\begin{gathered}
A_{m, k, n}=\operatorname{tr}\left(\varphi_{n} \psi_{k} \varphi_{m}^{\dagger}\right), \\
B_{m, k, n}=\operatorname{tr}\left(\varphi_{n} \varphi_{m}^{\dagger} \psi_{k}\right), \\
C_{m, k, n}=\operatorname{tr}\left(\varphi_{n} \varphi_{m} \psi_{k}\right) .
\end{gathered}
$$

Note that the above coefficients are related to the dissipator via its eigenstates $\left\{\psi_{k}\right\}$ and the associated biorthogonal basis $\left\{\varphi_{k}\right\}$. For a nondegenerate eigenvalue, deg $=1$, only the simplified operators $U_{k, k}=U_{k}$ and $W_{k, k}=W_{k}$ appear in Eq. (8), where

$U_{k}=g_{0}+\sum_{n>0} B_{n, k, k} g_{n}^{\dagger}, \quad W_{k}=g_{0}+\sum_{n>0} A_{n, k, k} g_{n}^{\dagger}$.

Equation (8) applies also in the presence of more degenerate eigenvalues.

The above statement follows from a perturbative Dyson expansion with respect to the small parameter $1 / \Gamma$ of the Liouvillian equation for $\rho(t)$, where $t$ is the rescaled time $t=\Gamma \tau$. With this scaling, we have $d \rho(t) / d t=\mathcal{L}_{0}[\rho(t)]+K[\rho(t)]$, where $\mathcal{L}_{0}[\cdot]=\mathcal{D}[\cdot] \quad$ and 
$K[\cdot]=-(i / \Gamma)[H, \cdot]$. The corresponding exact propagator, namely, $\exp \left[\left(\mathcal{L}_{0}+K\right) t\right]$, can be expanded in a Dyson series with respect to the perturbation $K$. Keeping the expansion terms up to order $K^{2}$ included and coming back to the time $\tau$, after some algebra, we get Eq. (8). Full details of the proof are given in Supplemental Material [21].

Eigenvalues and eigenvectors of $\mathcal{L}$. - By finding the normal modes of the linear problem (8) for each index $k>0$, as well as of the linear problem (2) for $k=0$, we obtain all the eigenvalues of the Liouvillian $\mathcal{L}$. Let $\lambda_{k, \alpha, \beta}$ be the set of the eigenvalues of $\mathcal{L}$ corresponding to $c_{k}$. First consider a nondegenerate $c_{k}$. In the limit $\Gamma \rightarrow \infty$, the $O(1 / \Gamma)$ contributions in Eq. (8) can be neglected and, expanding $R_{k}(\tau)=\sum_{\alpha, \beta} c_{\alpha \beta}(\tau)|\alpha\rangle\langle\tilde{\beta}|$, where $|\alpha\rangle$ are the right eigenvectors of $U_{k}$ with eigenvalues $u_{\alpha}$ and $\langle\tilde{\beta}|$ are the left eigenvectors of $W_{k}$ with eigenvalues $w_{\beta}$, we find $\quad d c_{\alpha \beta}(\tau) / d \tau=\lambda_{k, \alpha, \beta} c_{\alpha \beta}(\tau)=\left[c_{k} \Gamma+i\left(u_{\alpha}-w_{\beta}\right)\right] c_{\alpha \beta}(\tau)$. This implies

$$
\lambda_{k, \alpha, \beta}=c_{k} \Gamma+i\left(u_{\alpha}-w_{\beta}\right)+O(1 / \Gamma),
$$

with corresponding eigenvectors $\psi_{k, \alpha, \beta}=\psi_{k} \otimes|\alpha\rangle\langle\tilde{\beta}|$. Note that, even if not explicitly indicated, the eigenvalues $w_{\alpha}$ and $u_{\beta}$ depend, as the corresponding eigenvectors do, on the index $k$.

The $1 / \Gamma$ corrections to the eigenvalues (17) are then found by a standard perturbative formula, $\delta \lambda_{k, \alpha, \beta}=$ $\left\langle R_{\alpha \beta}\left|\hat{V}_{k}\right| R_{\alpha \beta}\right\rangle$, where $\hat{V}_{k}$ is the vectorized form of the $O(1 / \Gamma)$ term in the superoperator of Eq. (8) and $\left|R_{\alpha \beta}\right\rangle$ is an eigencomponent of the reduced density matrix $\left|R_{\alpha \beta}\right\rangle=|\alpha\rangle \otimes|\tilde{\beta}\rangle^{*}$. Every perturbative term of type $Q|x\rangle\langle y| P$ in (8) gives a contribution $\operatorname{tr}(Q|x\rangle\langle y|P| y\rangle\langle x|)=$ $\langle x|Q| x\rangle\langle y|P| y\rangle$ to the eigenvalue correction. Explicitly, we obtain

$$
\begin{aligned}
\delta \lambda_{k, \alpha, \beta}= & \frac{1}{\Gamma} \sum_{z>0} \sum_{m>0} \sum_{n: c_{n} \neq c_{k}} \frac{1}{c_{n}-c_{k}}\left(-\gamma_{m, z}^{n, n, k}\left\langle\alpha\left|g_{m}\right| \alpha\right\rangle\right. \\
& \left.\times\left\langle\tilde{\beta}\left|g_{z}^{\dagger}\right| \tilde{\beta}\right\rangle+\varepsilon_{z, m}^{n, n, k}\left\langle\alpha\left|g_{z}^{\dagger} g_{m}\right| \alpha\right\rangle+\delta_{z, m}^{n, n, k}\left\langle\tilde{\beta}\left|g_{z}^{\dagger} g_{m}\right| \tilde{\beta}\right\rangle\right) .
\end{aligned}
$$

The $O(1 / \Gamma)$ corrections to the respective eigenstates $\psi_{k} \otimes$ $|\alpha\rangle\langle\tilde{\beta}|$ are also given by standard first-order perturbative formulas [22].

Of course, the above $1 / \Gamma$ correction is valid if the eigenvalues (17) are nondegenerate. In the case of $\lambda_{k, \alpha, \beta}$ degenerate, a different, although still standard, procedure must be undertaken (diagonalization within the subspace of degeneration) to obtain the $1 / \Gamma$ corrections. Explicit expressions will be given for the case study considered below.

The case of a degenerate dissipator eigenvalue $c_{k}$ can be tackled in a similar way.
A case study: The XYZ spin chain.-We illustrate the above results on a Heisenberg spin chain with $N+1$ sites, the first one being in contact with a strongly dissipative environment. The coherent part of the evolution is given by the standard $X Y Z$ Hamiltonian $H=\sum_{j=0}^{N-1} \sum_{\alpha=x, y, z} \sigma_{j}^{\alpha} J_{\alpha} \sigma_{j+1}^{\alpha}, \quad \sigma_{j}^{\alpha}$ being the $\alpha$ th Pauli matrix acting at site $j$, whereas dissipation acts locally on site 0 and targets an arbitrary, pure or mixed, single spin state $\rho_{0}$ at this site [23]. The evolution of the density matrix $\rho(\tau)$ of the full chain is determined by a LME with Liouvillian as in Eq. (1). The Lindblad dissipator acting on spin 1 is the sum of two terms, $\mathcal{D}=[(1+\mu) / 2] \mathcal{D}_{1}+[(1-\mu) / 2] \mathcal{D}_{2}$,

$\mathcal{D}_{\alpha}[\rho]=L_{\alpha} \rho L_{\alpha}^{\dagger}-\frac{1}{2} L_{\alpha}^{\dagger} L_{\alpha} \rho-\frac{1}{2} \rho L_{\alpha}^{\dagger} L_{\alpha}, \quad \alpha=1,2$,

where $\quad L_{1}=|s(\theta, \varphi)\rangle\left\langle s^{\perp}(\theta, \varphi)\right| \quad$ and $\quad L_{2}=L_{1}^{\mathrm{T}}$, with $|s(\theta, \varphi)\rangle=\cos (\theta / 2) e^{-i \varphi / 2}|\uparrow\rangle+\sin (\theta / 2) e^{i \varphi / 2}|\downarrow\rangle$ and $\left\langle s(\theta, \varphi) \mid s^{\perp}(\theta, \varphi)\right\rangle=0$. This dissipator targets the polarization $\mu \vec{n}_{0}$ on site 0 , where $\vec{n}_{0}$ is the unit vector $\vec{n}_{0}=(\sin \theta \cos \varphi, \sin \theta \sin \varphi, \cos \theta)$. The uniqueness of the nonequilibrium stationary state (NESS) can be proven using Evans criterion [24].

Striped structure of spectrum.-The distribution of the Liouvillian eigenvalues manifestly depends on the strength of dissipation $\Gamma$ in Eq. (1). For medium dissipation strengths, comparable with the exchange integral in the model, the eigenvalues are scattered seemingly randomly, see Fig. 1 (top). For large $\Gamma$, they are arranged in distinct stripes, see Fig. 1 (bottom). The stripelike structure stems from the properties of the dissipator in the LME. In fact, the eigenvalue problem (3) of the locally acting dissipator $\mathcal{D}$ can be easily solved [18], yielding

$$
\begin{array}{ll}
c_{0}=0, & \psi_{0}=\frac{1+\mu}{2}|s\rangle\left\langle s\left|+\frac{1-\mu}{2}\right| s^{\perp}\right\rangle\left\langle s^{\perp}\right|, \\
c_{1}=-\frac{1}{2}, & \psi_{1}=|s\rangle\left\langle s^{\perp}\right|, \\
c_{2}=-\frac{1}{2}, & \psi_{2}=\left|s^{\perp}\right\rangle\langle s|, \\
c_{3}=-1, & \psi_{3}=|s\rangle\left\langle s|-| s^{\perp}\right\rangle\left\langle s^{\perp}\right|,
\end{array}
$$

where $|s\rangle \equiv|s(\theta, \varphi)\rangle$. The respective biorthogonal basis $\left\{\varphi_{k}\right\}$ is given by

$$
\begin{aligned}
\varphi_{0} & =I_{\mathcal{H}}, \quad \varphi_{1}=\psi_{2}, \quad \varphi_{2}=\psi_{1}, \\
\varphi_{3} & =\frac{1-\mu}{2}|s\rangle\left\langle s\left|-\frac{1+\mu}{2}\right| s^{\perp}\right\rangle\left\langle s^{\perp}\right| .
\end{aligned}
$$

Neglecting the coherent part provided by the Hamiltonian $H$, the Liouvillian $\mathcal{L}$ of Eq. (1) would have the eigenvalues $\Gamma c_{k}, k=0, \ldots, 3$, each eigenvalue having a degeneracy $2^{2 N}$ due to the inclusion of the $N$ extra spins. Adding $H$ acts as a 


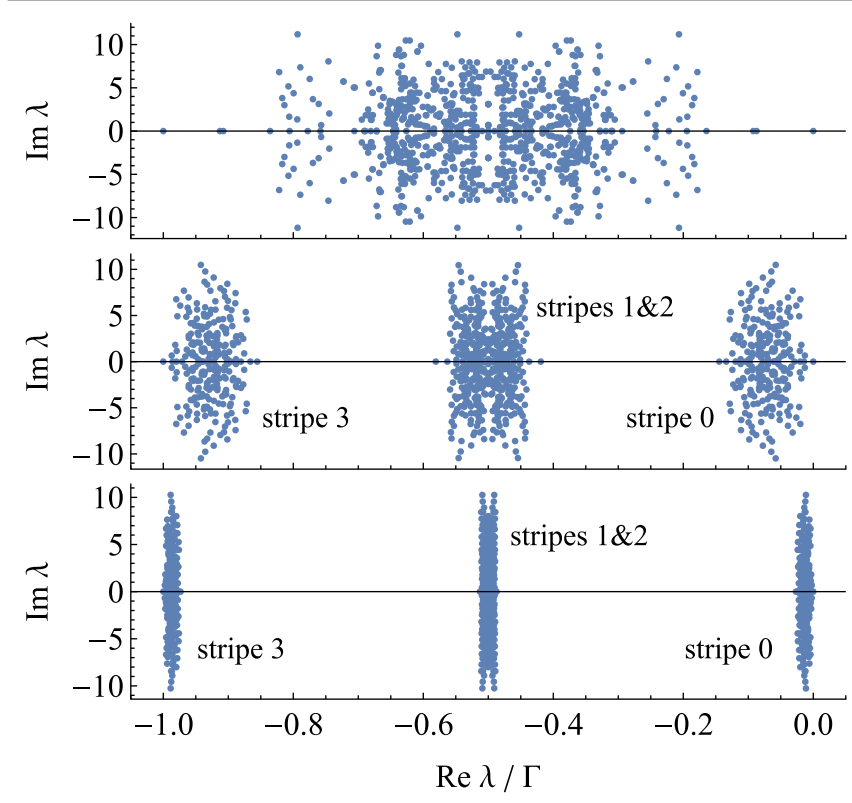

FIG. 1. Exact complex eigenvalues of the Liouvillian evaluated numerically for $\Gamma=0.5,8,20$ (from top to bottom). Increasing $\Gamma$, eigenvalues arrange in stripes whose number equals that of the eigenvalues of the dissipator. For $\Gamma$ large, the width of the stripes scales as $1 / \Gamma$ while their height remains constant; the distance between the stripes scales as $\Gamma$. Parameters: $N=4$, $\vec{J}=(1,1,-0.6058), \varphi=0, \theta=\pi / 2, \mu=1$.

perturbation (the small parameter being $1 / \Gamma$ ), which results in lifting the degeneracies. The perturbation-affected eigenvalues have, therefore, real part approximately given by $\Gamma c_{k}$.

Spectra of the stripes.-The stripe associated with $c_{0}=0$ is described by the equation for $R_{0}$ obtained in $[18,25]$. We review this equation and evaluate the corresponding spectrum in [21]. The other Zeno stripes are associated with the nonzero eigenvalues of the dissipator (20): $c_{1}=c_{2}=-1 / 2$ and $c_{3}=-1$. In the following, we consider the eigenvalue $c_{3}$. The analysis of the degenerate eigenvalue $c_{1}=c_{2}$ is similar and detailed in [21].

To evaluate the $O(1)$ terms of Eq. (8) for $k=3$, we need the operators $U_{3}$ and $W_{3}(16)$. The only nonzero coefficients $A_{n, 3,3}$ and $B_{n, 3,3}$ with $n>0$ are $A_{3,3,3}=B_{3,3,3}=-\mu$ and we find $U_{3}=W_{3}=g_{0}-\mu g_{3}^{\dagger}=\sum_{j=1}^{N-1} h_{j, j+1}-\mu\left(J \vec{n}_{0}\right) \cdot \vec{\sigma}_{1}$, where $h_{j, j+1}=\vec{\sigma}_{j} \cdot\left(J \vec{\sigma}_{j+1}\right)$ is the local density of the Hamiltonian $H$. Comparing $U_{3}$ and $h_{D}$, we see that they differ just by the sign of the local field acting on site 1. It can be shown that $h_{D}$ and $U_{3}$ are, therefore, isospectral [21]. According to (17), the corresponding Liouvillian eigenvalues are

$$
\lambda_{3, \alpha, \beta}=-\Gamma+i\left(\epsilon_{\alpha}-\epsilon_{\beta}\right)+O(1 / \Gamma),
$$

and the corresponding eigenvectors are $\psi_{3, \alpha, \beta}=\left|\psi_{3}\right\rangle \otimes$ $|\alpha\rangle\langle\beta|+O(1 / \Gamma)$, where $|\alpha\rangle$ is an eigenvector of $U_{3}$ with eigenvalue $\epsilon_{\alpha}$. The corrections $O(1 / \Gamma)$ are evaluated

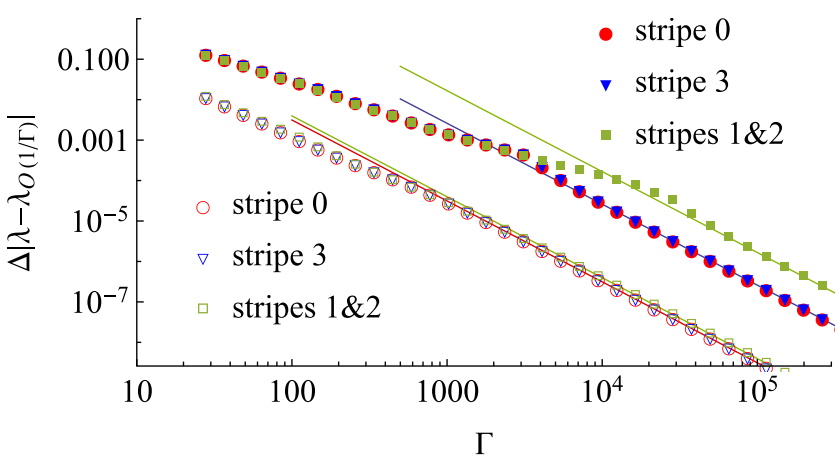

FIG. 2. Standard deviation of the modulus of the difference between numerically obtained Liouvillian eigenvalues and our perturbative prediction as a function of $\Gamma$, separately for each stripe. The set of data corresponding to empty symbols is obtained with parameters as in Fig. 1 (pure target state). The set with filled symbols corresponds to a mixed target state with parameters: $\quad N=4, \vec{J}=(1,1.7,-0.137), \quad \varphi=0, \quad \theta=2 \pi / 7$, $\mu=-0.7$. The straight lines are $\left(\Gamma_{c} / \Gamma\right)^{2}$ with, from top to bottom, $\Gamma_{c}=129,51,6.3,5.6$.

according to Eq. (18) for $\beta \neq \alpha$. The case $\beta=\alpha$ is similar to the calculation done for $c_{0}=0$ and is detailed in [21].

Figure 2 shows, stripe by stripe, the standard deviation of the error obtained by comparing the numerically computed Liouvillian eigenvalues with our perturbative eigenvalues, order $O(1 / \Gamma)$ included. As expected, this error behaves like $\left(\Gamma_{c} / \Gamma\right)^{2}$ for $\Gamma$ sufficiently large, with $\Gamma_{c}$ possibly different for the various stripes depending on the parameters chosen. The value of $\Gamma_{c}$ can be used as an indicator of an onset of the Zeno regime, characterized by the appearance of stripes in Fig. 1. From Fig. 2 we also see that the Zeno regime is reached easier for larger boundary gradient $|\mu|$.

Our Zeno-limit expansion for an eigenvalue $\lambda$ is applicable if the dissipation $\Gamma$ is much larger than the inverse radius of convergence of the $1 / \Gamma^{k}$ perturbative series for $\lambda$. The global radius of convergence, valid for all Liouvillian eigenvalues, is problem specific. In Fig. 3 we show, as a function of $\Gamma$, the real part of all Liouvillian eigenvalues of a Heisenberg chain with 2 spins, the first spin being targeted by a $z$-polarizing dissipation. Depending on the anisotropy, we find up to eight exceptional points, where two or more eigenvalues coalesce [26-29]. Fully analytical Zeno regime sets in beyond the rightmost branching point, see Fig. 3.

Let us summarize our findings. The eigenvalues of a Liouvillian with a locally acting dissipator at large dissipation strength $\Gamma$ are arranged in a set of stripes, see Fig. 1, indicating the existence of a hierarchy of relaxation timescales in the system [30]. The number of stripes coincides with the number of different eigenvalues of the Lindblad dissipator $\mathcal{D}$ in (1).

The width of the stripes scales as $1 / \Gamma$ and the distance between the stripes scales as $\Gamma$. The vertical extension of the 


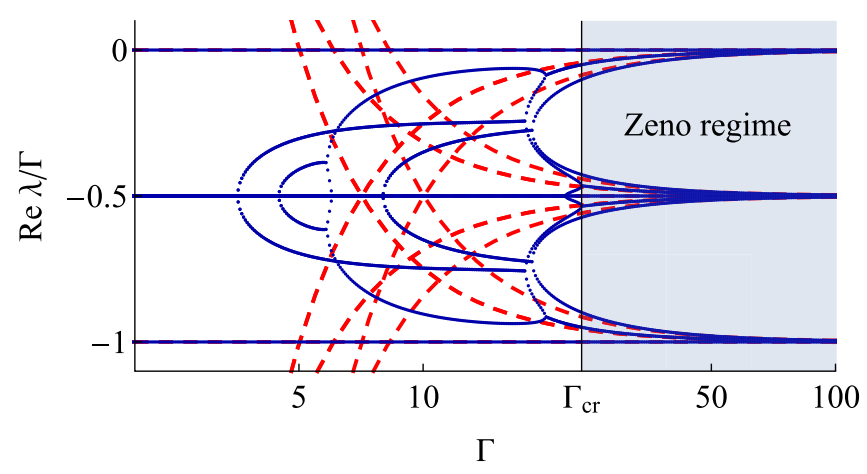

FIG. 3. Rescaled real part of all Liouvillian eigenvalues versus $\Gamma$, for $N=1$. Parameters: $\vec{J}=(1,2.3,-0.61), \varphi=\theta=0$, and $\mu=1$. Dashed lines show the near Zeno-limit predictions detailed in [21]. The vertical line at $\Gamma_{\text {cr }}$ marks the location of the rightmost branching points where Zeno regime sets in.

stripes does not depend on $\Gamma$ and is of the order of the norm $\|H\|$ of the coherent part of the Liouvillian (1). The position of the stripes on the real axis is $\operatorname{Re} \lambda=c_{k} \Gamma+O(1 / \Gamma)$ where $c_{k}$ are the eigenvalues of the dissipator (3). Each stripe corresponding to a nondegenerate $c_{k}$ contains $d_{1}^{2}$ Liouvillian eigenvalues, where $d_{1}=\operatorname{dim} \mathcal{H}_{1}$ is the dimension of that part of Hilbert space which is not affected directly by the dissipation. Emergence of stripes can be viewed as a hallmark of a quantum Zeno regime.

We derived linear spectral problems for the dissipationprojected Liouvillian, for each relaxation mode $c_{k}$, and outlined a complete solution of the eigenvalue problem via a perturbative analysis. We demonstrated our general results in the case of dissipation acting on a single boundary qubit of an anisotropic Heisenberg spin chain. For this case, we obtained explicit expressions for eigenvalues and eigenvectors of the problem near the Zeno regime. The solutions are given in terms of spectral data of a dissipation-projected Hamiltonian and other similar Hamiltonians, these being much simpler objects than the original Liouvillian. Our method is straightforwardly applicable to the $X Y Z$ model with dissipation acting on both boundaries, thus creating boundary gradients [31,32], which play a prominent role in studies of quantum transport [33]. All the auxiliary Hamiltonians have the form of an open $X Y Z$ spin chain with boundary fields and are integrable [34].

To derive our results we used several assumptions: (i) diagonalizability of the dissipator (3), (ii) uniqueness of its kernel, (iii) absence of anomalous scaling of the gaps in the spectrum of the Liouvillian, including the Liouvillian gap. A generalization of our results is, in principle, straightforward. We expect the emergence of striped structure and scaling of the stripes in the Zeno limit to be qualitatively correct also for degenerate kernels, e.g., for those resulting from Hermitian Lindblad operators [35].
Our explicit results shed a light on the intrinsic properties of an isolated system coupled strongly to the environment, and make its study almost analytically affordable.

V.P. gratefully acknowledges financial support by the Deutsche Forschungsgemeinschaft through DFG project KL 645/20-1, by the European Research Council through the advanced Grant No. 694544-OMNES, and thanks the Department of Physics of Sapienza University of Rome for hospitality and financial support.

[1] V. Kendon, Quantum computing using continuous-time evolution, Interface Focus 10, 20190143 (2020).

[2] J. Cao et al., Quantum biology revisited, Sci. Adv. 6, eaaz4888 (2020).

[3] H.-J. Briegel and B.-G. Englert, Quantum optical master equations: The use of damping bases, Phys. Rev. A 47, 3311 (1993).

[4] S. M. Barnett and S. Stenholm, Spectral decomposition of the Lindblad operator, J. Mod. Opt. 47, 2869 (2000).

[5] D. Rocca, R. Gebauer, Y. Saad, and S. Baroni, Turbo charging time-dependent density-functional theory with Lanczos chains, J. Chem. Phys. 128, 154105 (2008).

[6] J. M. Torres, R. Betzholz, and M. Bienert, Optomechanical damping basis, J. Phys. A 52, 08LT02 (2019).

[7] M. Marcuzzi, J. Schick, B. Olmos, and I. Lesanovsky, Effective dynamics of strongly dissipative Rydberg gases, J. Phys. A 47, 482001 (2014).

[8] V. V. Albert, B. Bradlyn, M. Fraas, and L. Jiang, Geometry and Response of Lindbladians, Phys. Rev. X 6, 041031 (2016).

[9] O. Shpielberg, Diffusion and entanglement in open quantum systems, Europhys. Lett. 129, 60005 (2020).

[10] M. V. Medvedyeva, F. H. L. Essler, and T. Prosen, Exact Bethe Ansatz Spectrum of a Tight-Binding Chain with Dephasing Noise, Phys. Rev. Lett. 117, 137202 (2016).

[11] L. R. Bakker, V. I. Yashin, D. V. Kurlov, A. K. Fedorov, and V. Gritsev, Lie-algebraic approach to one-dimensional translationally invariant free-fermionic dissipative systems, Phys. Rev. A 102, 052220 (2020).

[12] B. Buca, C. Booker, M. Medenjak, and D. Jaksch, Bethe ansatz approach for dissipation: Exact solutions of quantum many-body dynamics under loss, New J. Phys. 22, 123040 (2020).

[13] M. Nakagawa, N. Kawakami, and M. Ueda, Exact Liouvillian Spectrum of a One-Dimensional Dissipative Hubbard Model, Phys. Rev. Lett. 126, 110404 (2021).

[14] B. Misra and E. C. G. Sudarshan, The Zeno's paradox in quantum theory, J. Math. Phys. (N.Y.) 18, 756 (1977).

[15] C. Presilla, R. Onofrio, and U. Tambini, Measurement quantum mechanics and experiments on quantum Zeno effect, Ann. Phys. (N.Y.) 248, 95 (1996).

[16] K. Koshino and A. Shimizu, Quantum Zeno effect by general measurements, Phys. Rep. 412, 191 (2005).

[17] D. Burgarth, P. Facchi, H. Nakazato, S. Pascazio, and K. Yuasa, Quantum Zeno dynamics from general quantum operations, Quantum 4, 289 (2020). 
[18] V. Popkov, S. Essink, C. Presilla, and G. M. Schütz, Effective quantum Zeno dynamics in dissipative quantum systems, Phys. Rev. A 98, 052110 (2018).

[19] P. Zanardi and L. Campos Venuti, Coherent Quantum Dynamics in Steady-State Manifolds of Strongly Dissipative Systems, Phys. Rev. Lett. 113, 240406 (2014).

[20] V. Gorini and A. Kossakowski, $N$-level system in contact with a singular reservoir, J. Math. Phys. (N.Y.) 17, 1298 (1976).

[21] See Supplemental Material at http://link.aps.org/supplemental/ 10.1103/PhysRevLett.126.190402 for details.

[22] L. D. Landau and E. M. Lifshitz, Quantum Mechanics: NonRelativistic Theory, 3rd ed. (Pergamon Press, Oxford, 1989).

[23] T. Prosen, Exact Nonequilibrium Steady State of a Strongly Driven Open $X X Z$ Chain, Phys. Rev. Lett. 107, 137201 (2011).

[24] D. E. Evans, Irreducible quantum dynamical semigroups, Commun. Math. Phys. 54, 293 (1977).

[25] V. Popkov, S. Essink, C. Kollath, and C. Presilla, Dissipative generation of pure steady states and a gambler's ruin problem, Phys. Rev. A 102, 032205 (2020).

[26] T. Kato, Perturbation Theory of Linear Operators (Springer, Berlin, 1966).

[27] E. J. Bergholtz, J. C. Budich, and F. K. Kunst, Exceptional topology of non-Hermitian systems, Rev. Mod. Phys. 93, 015005 (2021).
[28] W. D. Heiss, The physics of exceptional points, J. Phys. A 45, 444016 (2012).

[29] N. Hatano, Exceptional points of the Lindblad operator of a two-level system, Mol. Phys. 117, 2121 (2019).

[30] K. Wang, F. Piazza, and D. J. Luitz, Hierarchy of Relaxation Timescales in Local Random Liouvillians, Phys. Rev. Lett. 124, 100604 (2020).

[31] V. Popkov, T. Prosen, and L. Zadnik, Exact Nonequilibrium Steady State of Open $X X Z / X Y Z$ Spin-1/2 Chain with Dirichlet Boundary Conditions, Phys. Rev. Lett. 124, 160403 (2020).

[32] V. Popkov, T. Prosen, and L. Zadnik, Inhomogeneous matrix product ansatz and exact steady states of boundary-driven spin chains at large dissipation, Phys. Rev. E 101, 042122 (2020).

[33] B. Bertini, F Heidrich-Meisner, C. Karrasch, T. Prosen, R. Steinigeweg, and M. Znidaric, Finite-temperature transport in one-dimensional quantum lattice models, arXiv:2003 .03334

[34] Y. Wang, W.-L. Yang, J. Cao, and K. Shi, Off-Diagonal Bethe Ansatz for Exactly Solvable Models (Springer, New York, 2015), ISBN 978-3-662-46755-8.

[35] T. Can, V. Oganesyan, D. Orgad, and S. Gopalakrishnan, Spectral Gaps and Midgap States in Random Quantum Master Equations, Phys. Rev. Lett. 123, 234103 (2019). 